\title{
COST- EFFECTIVE APPROACH FOR 100GBPS VCSEL-MMF LAN'S
}

\author{
Rajat Bansal $^{1}$, Neetu Sharma ${ }^{2}$ \\ ${ }^{1}$ Student, ECE, BGIET, Punjab, India \\ ${ }^{2}$ Assistant Professor, ECE, BGIET, Punjab, India
}

\begin{abstract}
VCSELs enable many types of applications, such as optical computing and optical information processing. In this work, firstly different physical parameters Temperature, cavity volume and Bias current have been analysed on VCSEL laser. Further different modulation formats i.e. NRZ, CSRZ and MDRZ are analysed for the first time using VCSEL Laser over GI-MMF in terms of Qfactor and BER.MDRZ comes out to be best modulation formats to prolong the system reach to28Km. Performance analysis of 100 Gbps MIMO system using MDRZ modulation format over $28 \mathrm{~km}$ MMF has also been investigated at different distances varied from $8 \mathrm{~km}$ to $28 \mathrm{~km}$ in terms of $Q$-factor and BER.
\end{abstract}

Keywords: $M M F, G I, V C S E L, M D R Z, M I M O$

\section{INTRODUCTION}

Vertical Cavity surface-emitting lasers (VCSEL's) have been studied extensively over the past few years because of their useful characteristics such as a low threshold current, single-longitudinal-mode operation, circular output beam, and wafer-scale integrability [1], [2]. Since VCSEL's are attractive as compact light sources for applications in optical communications and interconnects, it is important to have a thorough understanding of their noise characteristics. Indeed, extensive experimental studies of the intensity-noise in VCSEL's have been performed [3]-[6]. Multimode fiber (MMF) links are widely implemented in current high-speed local area networks (LANs) [7]. Using VCSEL laser along with MMF provides a cost effective solution for metropolitan networks. They can provide the necessary bandwidth for shorter-length applications at much lower expense than single-mode fiber (SMF) solutions, mainly due to the ease of optical alignment and packaging. In MMF, however, the signal on each of the fiber modes propagates down the fiber with its own distinct velocity and thus causes Intersymbol interference (ISI). Recently, increased bandwidth-distance products have been demonstrated through the use of multiple-input multiple-output (MIMO) technology based on RF-over-fiber [8], [9], although reasonable performance is obtained only for long propagation distances which achieve the necessary optical delay spread. Multi-input multi-output (MIMO) communications over multi-mode fibers (MMFs) holds the promise of improving bandwidth efficiency. MIMO technology has aroused interest because of its possible applications in digital television (DTV), wireless local area networks (WLANs), metropolitan area networks (MANs), and mobile communications.

In this work, performance of VCSEL laser has been analysed for different physical parameters such as temperature, cavity volume and bias current. Work extended to investigate the combined effect of cavity volume on temperature to minimise performance fluctuations. Further, 10x10 MIMO system using different modulation formats over MMF using VCSEL has been observed at different distance and in terms of Q-factor and BER.

\section{Simulation Setup}

In this work performance analysis of 100 Gbps MIMO system over graded index multimode fiber upto length of 28 $\mathrm{km}$ has been proposed. The purposed system consists of 10 VCSEL lasers with electrical drive from DC bias generators followed by MDRZ transmitter. All the channels are combined with ideal power combiner. The signal from power combiner transmitter over MMF is as shown in Fig. 1. The purpose of power combiner is to combine all the signals from the transmitter section and send to MMF. The power splitter is used to split the signal into 10 equal powers. The signal from MMF then received by receiver section consisting of photodetector pin used for optical to electrical conversion having dark current of $10 \mathrm{nA}$ and responsivity $1 \mathrm{~A} / \mathrm{W}$ followed by Low pass Bessel filter used to remove noise from the signal. The low pass Bessel filter has cutoff frequency of $0.75 \mathrm{~Hz}$ then $3 \mathrm{R}$ regenerator used for reshaping, resampling and re amplification followed by BER Analyzer. BER analyser is kept at the receiver output to examine the eye diagram and Q-factor. 


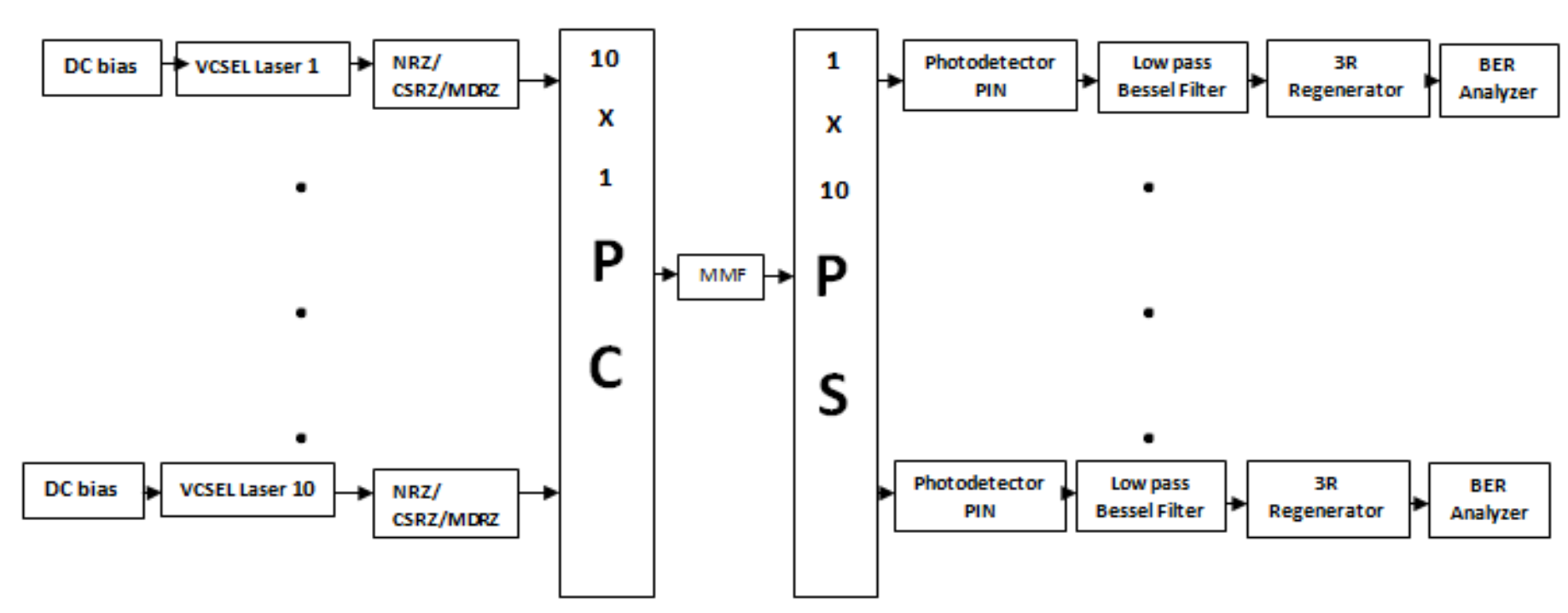

\section{Results and Discussion}

Results has been analysed for different physical parameters temperature, cavity volume and bias current using VCSEL Laser as shown in figure 2 (a), 2 (b) and 2(c). It is concluded that with increase in temperature, Quality of a signal decreases as shown in Figure 2(a). Similarly, it is also observed that with increase in bias current, quality of signal decrease shown in Figure 2(b) and further it is also calculated that with decrease in cavity volume the quality of the signal increases as shown in Figure 2(c).

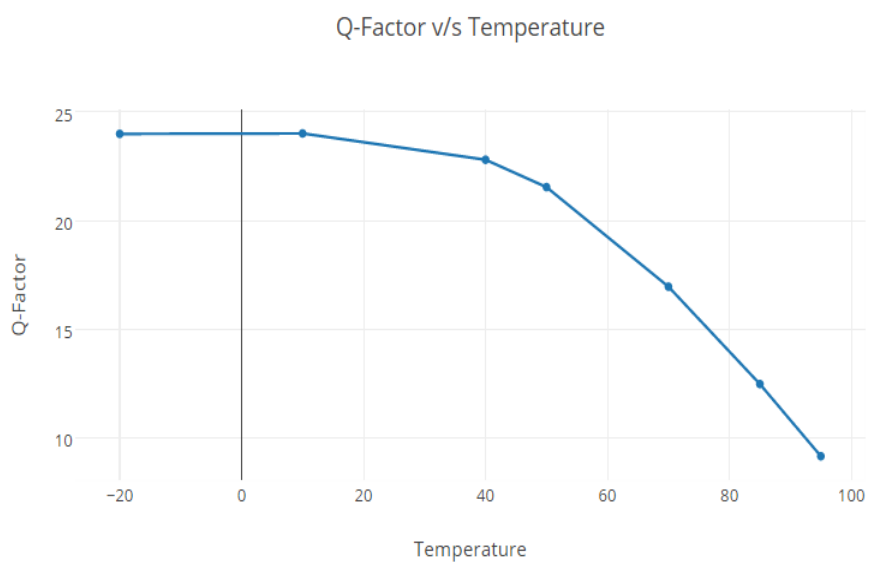

Figure 2(a) Q-factor v/s Temperature

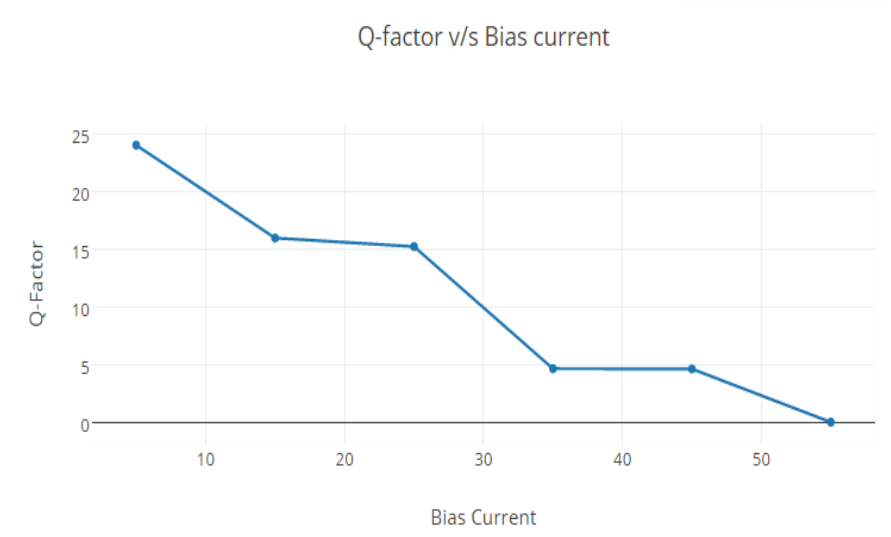

Figure 2(b) Q-factor v/s Bias Current

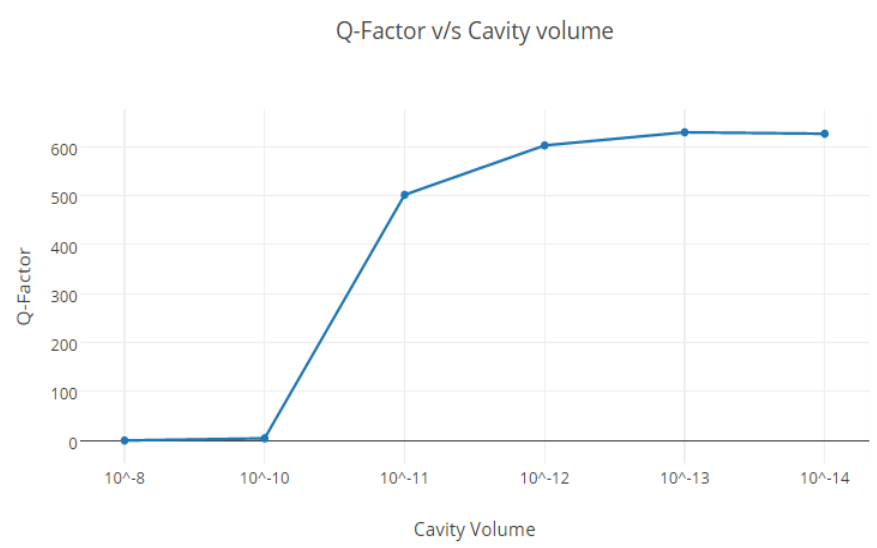

Figure 2(c) Q-factor v/s Cavity volume

Different modulation formats i.e. NRZ, CSRZ and MDRZ have been analysed. The length of MMF is varied from $400 \mathrm{~m}, 800 \mathrm{~m}, 1200 \mathrm{~m}, 1600 \mathrm{~m}, 2000 \mathrm{~m}$ and $2400 \mathrm{~m}$ and corresponding results are taken for each modulation format separately. The best results are shown by MDRZ modulation format as shown in Figure 3.

\section{Different modulation formats}

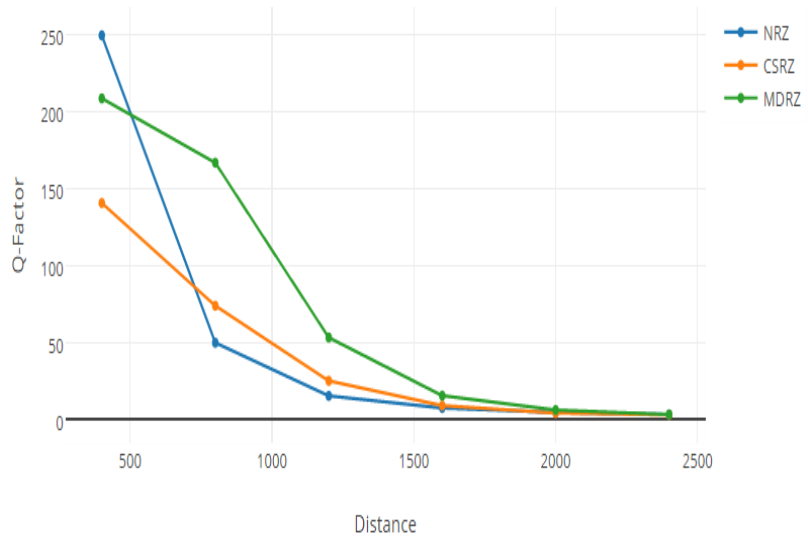

Figure 3: Comparison of Different Modulation formats 
The performance analysis of 100 Gbps MIMO system using MDRZ modulation format over $28 \mathrm{~km}$ MMF has been investigated at different distances varied from $8 \mathrm{~km}$ to 28 $\mathrm{km}$. Results has been analysed in terms of Q-factor and BER. It is observed that with the increase of transmission distance, performance of the system degrades as shown in Figure 4.

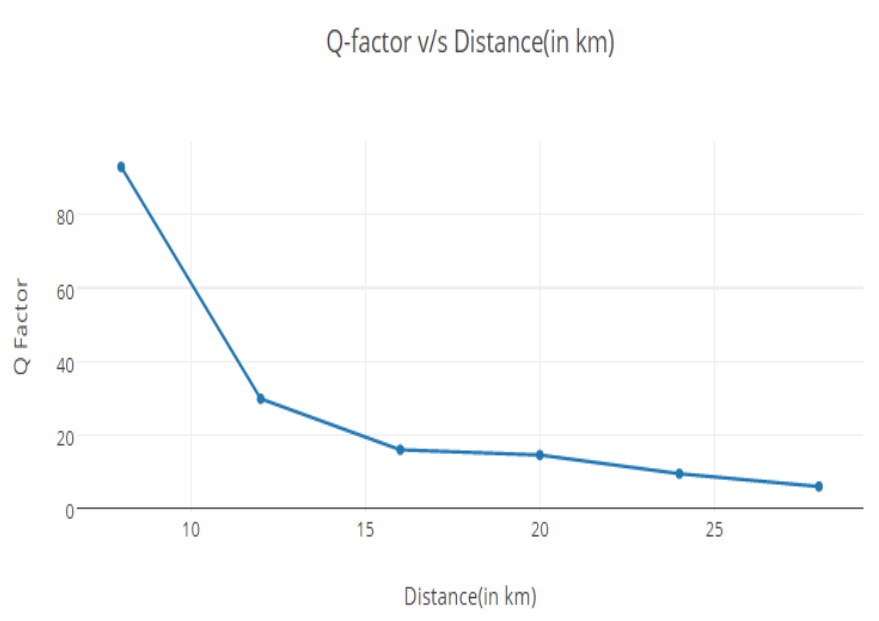

Figure 4: Q-factor v/s Distance (in km)

Figure 5 shows the Optical spectrum analyzer and Figure 6 shows the optical time domain visualizer. The eye diagram at minimum length $(8 \mathrm{~km})$ and maximum length $(28 \mathrm{~km})$ is shown in Figure 7(a) and 7(b) as follows.

룰

Optical Spectrum Analyzer

Dbl Click On Objects to open properties. Move Objects with Mouse Drag

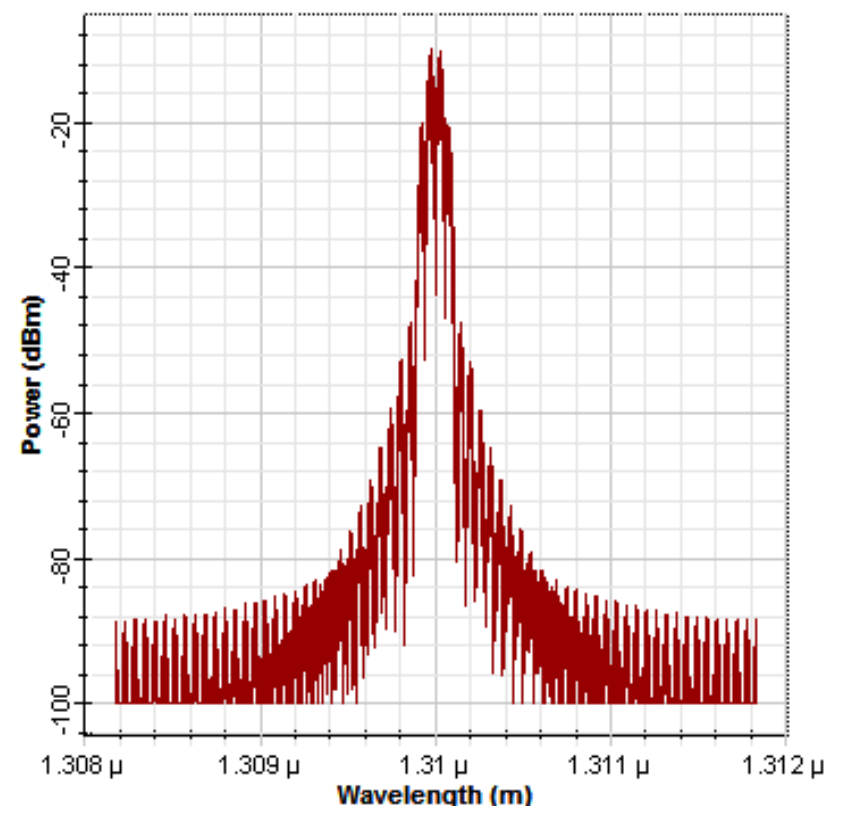

Figure 5: Optical spectrum analyzer

\section{兽 Optical Time Domain Visualizer_10}

Dbl Click On Objects to open properties. Move Objects with Mouse Drag

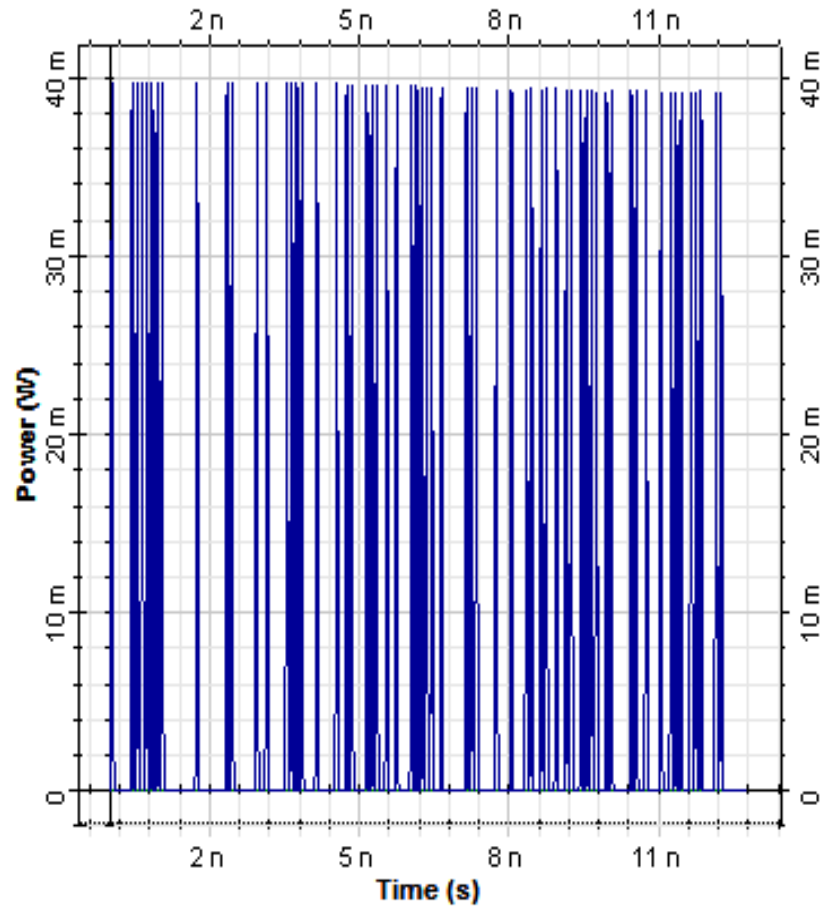

Figure 6: Optical time domain visualizer

苞 Dbl Click On Objects to open properties. Move Objects with Mouse Drag
Time (bit period)

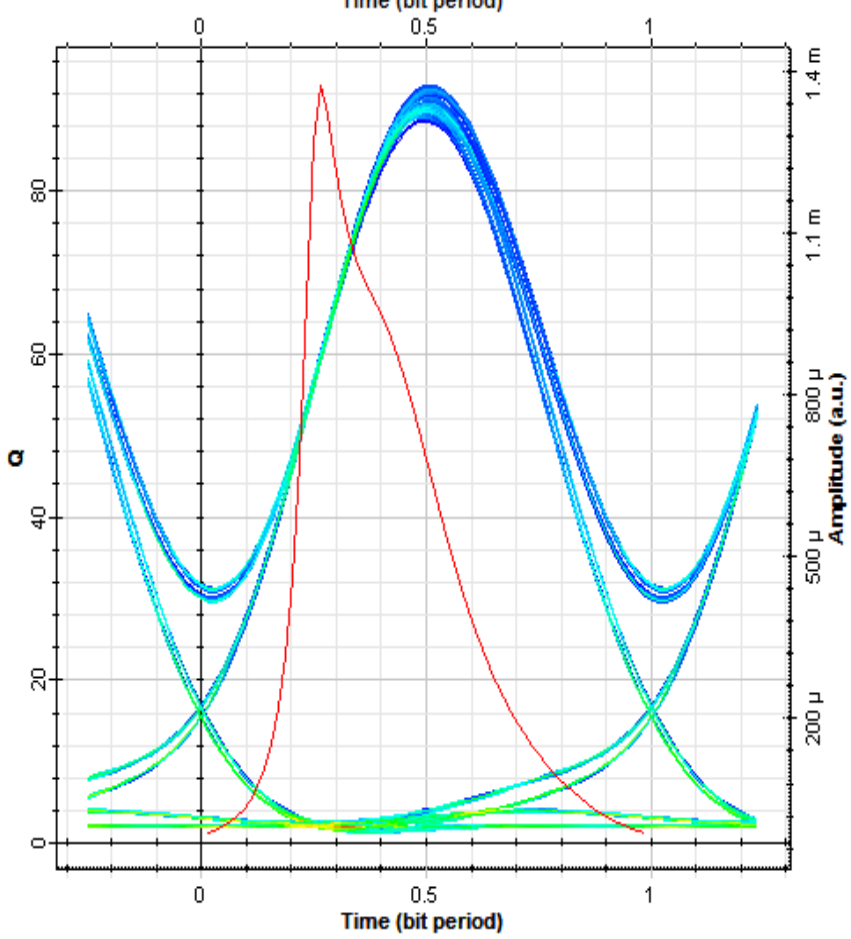

Figure 7(a): Length of fiber $8 \mathrm{~km}(\max )$ 


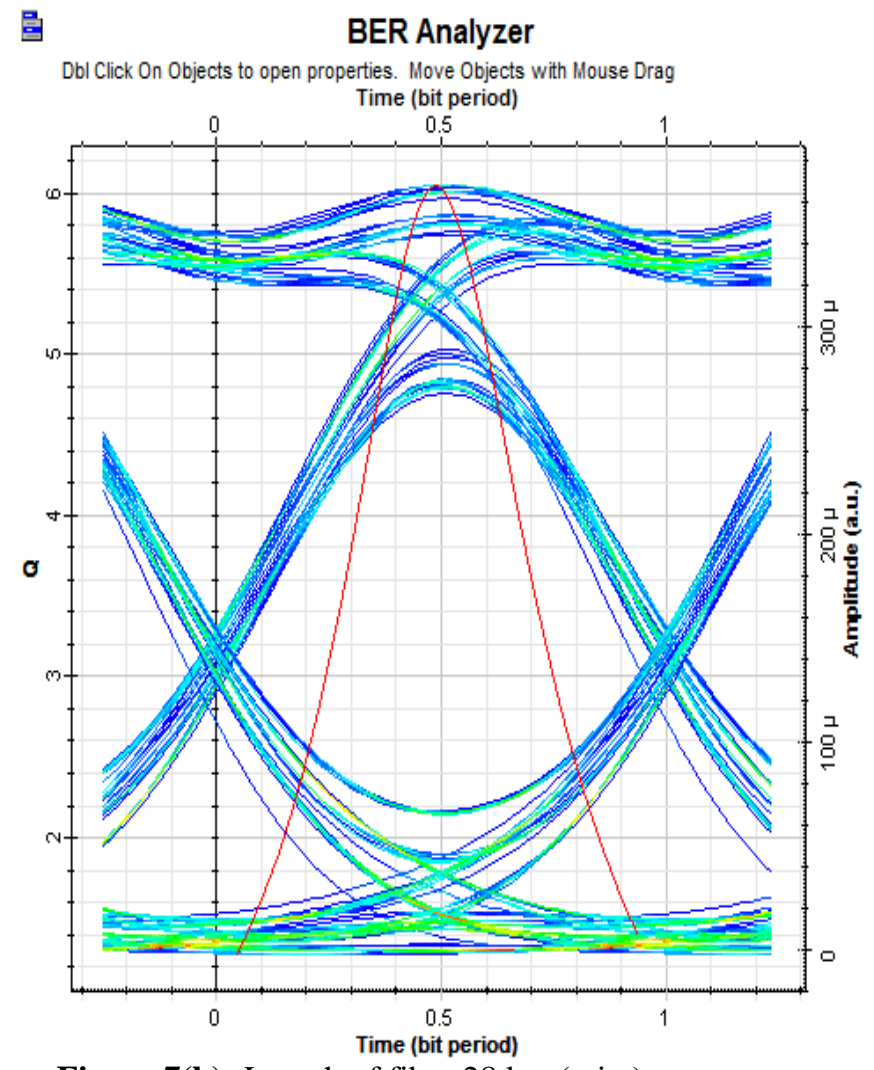

Figure 7(b): Length of fiber $28 \mathrm{~km}$ (min.)

Figure.7: Eye diagram showing fiber length (a) $8 \mathrm{~km}$ (b) 28 $\mathrm{km}$

\section{CONCLUSION}

We have analyzed the performance of VCSEL laser by varying different physical parameters like Temperature, cavity volume and bias current. It is observed that with the increase of temperature, Quality of the system decreases. Maximum performance has been reported at $5^{\circ} \mathrm{C}$ with Qfactor of 24.06 and worst for $95^{\circ} \mathrm{C}$ with Q-factor of 9.16 . Further we have investigated the combined effect of cavity volume and temperature. It is suggested that low cavity volume mitigate or overcome the effects of temperature on output power. Further, performance analysis of different modulation formats i.e. NRZ, CSRZ and MDRZ has been investigated. The length of MMF is varied from $400 \mathrm{~m}$, $800 \mathrm{~m}, 1200 \mathrm{~m}, 1600 \mathrm{~m}, 2000 \mathrm{~m}$ and $2400 \mathrm{~m}$ and corresponding results are taken for each modulation format separately. It is concluded that MDRZ modulation format shows the best result. Then, the performance analysis of 100 Gbps MIMO system using MDRZ modulation format over $28 \mathrm{~km}$ MMF has been investigated at different distances varied from $8 \mathrm{~km}$ to $28 \mathrm{~km}$. It is observed that with the increase of transmission distance, performance of the system degrades.

\section{REFERENCES}

[1] C. J. Chang-Hasnain, Semiconductor Lasers: Past, Present and Future, G. P. Agrawal, Ed. Woodbury, NY: AIP, 1995, ch. 5.

[2] C. J. Chang-Hasnain, J. P. Harbison, G. Hasnain, A. C. Von Lehmen, L. T. Florez, and N. G. Stoffel, "Dynamic, polarization and transverse mode characteristics of VCSELs,” IEEE J. Quantum Electron., vol. 27, pp. 1402-1408, 1991.

[3] D. M. Kuchta, J. Gamelin, J. D. Walker, J. Lin, K. Y. Lau, and J.S. Smith, "Relative intensity noise of vertical cavity surface emitting lasers," Appl. Phys. Lett., vol. 62, 1194-1196, 1993.

[4] M. S. Wu, L. A. Buckman, G. S. Li, K. Y. Lau, and C. J. Chang-Hasnain, "Polarization induced enhancement of relative intensity noise

and modulation distortion in vertical cavity surface emitting lasers," in IEEE 14th Int. Semiconductor Laser Conf., 1994, pp. 145-146.

[5] K. H. Hahn, M. R. Tan and S. Y. Wang, "Intensity noise of large area vertical cavity surface emitting lasers in multimode optical fiber links," Electron. Lett., vol. 30, pp. 139-140, 1994.

[6] K. H. Hahn, M. R. Tan, Y. M. Houng, and S. Y. Wang, "Large area multitransverse-mode VCSEL's for modal noise reduction in multimode fiber systems," Electron. Lett., vol. 20, pp. 1482-1483, 1993.

[7] IEEE Std. 802.3ae, “10 Gigabit Ethernet, 2002.

[8] H. R. Stuart, "Dispersive multiplexing in multimode fiber," in Proc. IEEE Optical Fiber Communication Conf., vol. 3, Baltimore, MD, Mar. 3-10 2000, pp. 305-307.

[9] Michael A. Jensen, "Dispersive multiplexing in multimode optical fiber," Science, vol. 289, pp. 281-283, Jul. 2000. 